\title{
A Uniform Control Interface for Various Electronic Aids
}

\author{
Christian Bühler, Helmut Heck, Rainer Wallbruch \\ Forschungsinstitut Technologie-Behindertenhilfe, Evangelische Stiftung Volmarstein \\ D-58300 Wetter, Germany
}

\begin{abstract}
The operation of technical aids by disabled and elderly people leads to enormous difficulties because users have to deal with different control philosophies and user interface concepts for each technical aid used. Therefore a point of general interest is not only the pure support function of the aid itself, but especially its human machine interface and the interdependencies with further aids used by the same handicapped person. A computer-based user interface management system is presented that allows an assistive person to individually tailored a human computer interface to the user's needs and capabilities which allows the handicapped user to operate very different devices by the same principle and mechanism of control.
\end{abstract}

\section{Computers for People with Special Needs}

People with disabilities are often not able to use standard computer hardware and software. Due to various impairments, e.g. physical impairment, mental retardation, or perceptual disorders, they cannot use normal keyboards and pointing devices or they are analphabets or they cannot read text on a monitor screen. But just for handicapped people a computer could open areas of their social and occupational environment. To make the problems clear: what can someone do, who is sitting in a wheelchair, unable to speak and write, and intending to switch on a light or a special TV channel. He can communicate by pointing at pictures (if his communication partner has plenty of practice with this) and ask someone to help him, or he can use a special switch which he can reach from the wheelchair. Both possibilities can be aided by a computer. A computer could aid communication, manage devices in the personal environment, could control a wheelchair or a robot arm. In the European TIDE (Technology Initiative for Disabled and Elderly People) programme $[1,2]$ the need to integrate systems has been broadly introduced.

Thus, handicapped people have special needs in operating computers $[3,4]$. This comprises not only input and output devices, but also the way of presenting audiovisual information and the complexity/abstraction of information [5]. It is desirable to have aiding devices that help those persons to overcome their handicaps but on the other hand involve as much of the mental, motor and sensoric capabilities of the user as possible. A computer gives the chance to build very individual user interfaces to control the functionality of the computer itself and of external devices. 


\section{Interface Problems}

The normal situation, when a user wants to work with different devices, is generally known. Each device has its own user interface and its own input devices (fig. 1a) [6].

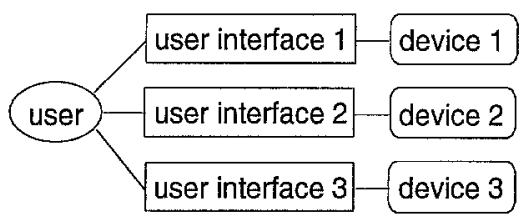

(a)



(b)

Fig. 1. Standard (a) versus optimal (b) situation when an user works with different devices

For handicapped persons this can mean a new problem: Often it is not possible to use different devices at the same or nearly at the same time. If someone drives a wheelchair he cannot use a communication aid at the same time, if the only movement he is able to perform is needed for driving the wheelchair. Usually a special device or switch is needed additionally to the wheelchair equipment and it is questionable if this could be operated then. Desirable is one user interface for various devices (fig 1b). For example, a communication aid and a powered wheelchair can be operated via the same input devices and the same monitor program.

In practical use of assisting devices, we encountered another interface problem: in the training situation of handicapped persons in our rehabilitation centre. As described above, the choice of input devices and the electronic aids themself depend on the abilities and needs of the handicapped persons. Every user has his own devices with specific interfaces. This means for a trainer, who e.g. wants to train persons in communicating via symbols, that he has to learn the operation and method of adaption (configuration) of all the communication devices in use. Furthermore, the persons in charge in a residential home for handicapped persons have lo learn all services and all the interfaces they have contact to (fig. 2a).

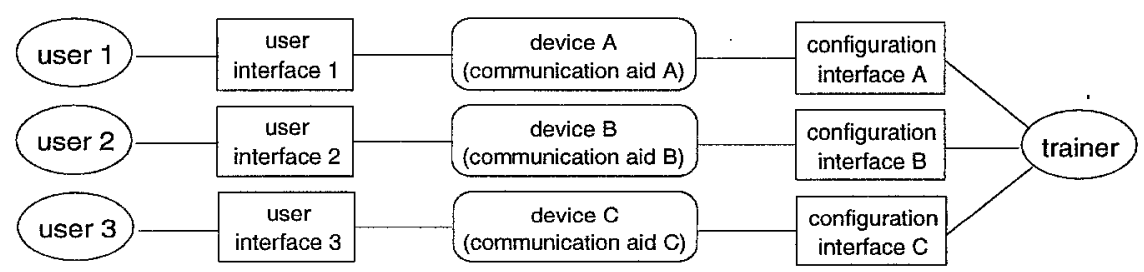

Fig. 2a. Standard situation when a trainer trains several users on devices of the same kind

Additionally, this is not only an inter-individual but also an intra-individual problem: in the case of assisted communication it is possible that someone who begins with a communication aid based on symbols learns more symbols or more complex symbol systems, or he learns to read and to write letters. Seldom the functionality of the communication aid grows as the abilities of its user grows. Usually the disabled person and also his trainer has to change to another 
communication aid, probably with a different user interface and with another configuration interface.

We found out that the great variety of assistive electronic devices with different human machine interface concepts means a crucial problem for all users, even more for the training staff (occupational therapists, teachers etc.) than for the disabled persons. Often much of the functionality of devices is not used because trainers are not able to or do not have enough time to adapt user interfaces to the users' needs. Here it is desirable, too, that the trainer has only one configuration interface for different devices (fig. 2b) or, respectively, a very flexible system with different user interfaces (fig. 2c).

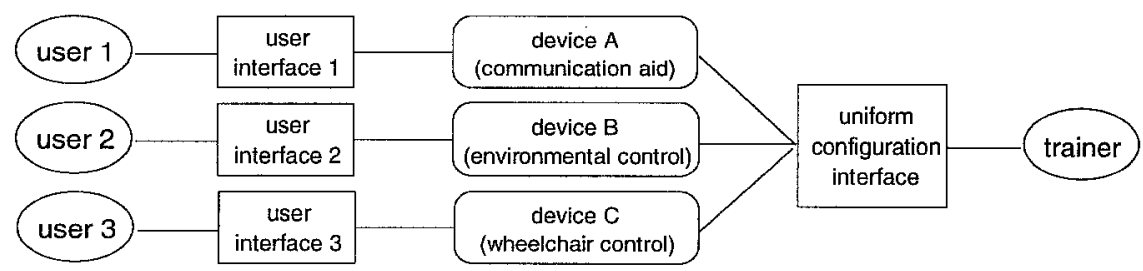

Fig. 2b. Optimal situation when a trainer trains several users on several devices

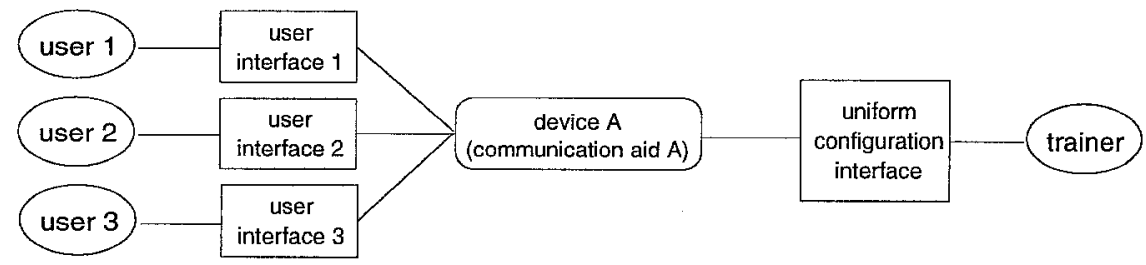

Fig. 2c. Optimal situation when a trainer trains several users on one device

\section{Demand for User Tailorable Systems}

From the situation described above, we formulated the demand for a versatile user interface management system (UIMS) for physically / mentally / perceptionally disabled people and their caring persons.

Uniform user interface. From the point of view of a disabled person, the UTMS should enable a very special, individually adapted and uniform user interface with - an individual input device out of a variety of switches, pointing devices, keyboards etc.,

- an individual presentation of information on the monitor screen out of a variety of pictures, symbols, texts, colours, windows, menues etc.,

- an individual selection/activation method of choices in menues from a variety of methods (direct selection, single-item scanning, row-column scanning etc.). 
suitable for the use of several different electronic aids (the computer included) at the same time. To the user a created user interface should appear uniform (with respect to operation) for all applications.

Uniform configuration interface. From the point of view of a training person, the UIMS should have one uniform configuration interface for all the different options of user interfaces and electronic aids.

\section{Implementation Model of the UIMS}

Figure 3 shows the implementation model of the UIMS. In correspondence with the different users, the trainer configures via the uniform configuration interface individual user interfaces, each of which gives access to different devices in a uniform way of operation.



Fig. 3. Implementation model of the UIMS

\section{The Configuration Interface}

The configuration interface provides the tools for the description of personal configurations for different users. This comprises:

- the choice of input and output devices,

- the way of presentation of the pictures/symbols/text on the screen

- choice of arbitrary symbols, pictures, scanned photographs or free-hand drawings,

- size of the symbols,

- order on the screen,

- colour of the background and the high-lighting, 
- display of text strings related to the symbols,

- combination of related symbols,

- organisation of menus,

- the mode of selection of symbols (direct, different ways of scanning),

- timing restrictions for activation of symbols (minimal duration, bolting)

- the assignment of speech output to symbols,

- the assignment of actions to symbols.

Furthermore the inclusion of new pictures/symbols and spoken words or sentences is supported. Necessary configuration input devices are the standard input devices as well as a microphon, a scanner (for scanning photographs or hand-drawn pictures) or an additional PC. According to the requirement that a communication aid has to be adaptable to different kinds of physical impairment, the user interface management system is able to cope with arbitrary input devices, like buttons, switches or pointing devices (mouse, trackball, joystick, touchscreen). Pictures or symbols to be selected are displayed on a colour monitor screen.

\section{Applications}

Symbol-based communication aids. In the following, examples of user interfaces for various areas are described. At first the case of a basic communication aid for nonspeaking persons [7], e.g. young children, is considered. Because the children cannot read and write, the input is based on graphical symbols, which are monitored on a flat screen and accessed by input devices according to the children's abilities

The delivered objects of the system, here graphical symbols, are in one of three states (passive, selected, activated). A state can be indicated on the screen by several variables: the colour of the symbols or the colour of the background can change, for instance. If a picture is selected, directly or by scanning, it can be activated by a switch and then it can produce two types of actions and a message for output. To each talking picture a speech output is assigned. If a talking picture is activated, a corresponding message is sent to the speech output control and the output, preferrable a digitised natural voice for this kind of restricted communication, follows directly. It is also possible to list at first a series of talking pictures in order to build a sentence with several statements and then select and activate a silent picture in the very same manner, which triggers the speech output.

Figure 4a shows an example of the screen. A statement composition line is found at the top. Beyond this, one window allows the selection of pictures according to a prior chosen theme. Another small window allows to go to wheelchair or environment control. The selection mode is direct, selection is indicated by a little arrow and a different background colour. Seen in the window on top, the chosen communication theme is "clothes" (selected and activated in the first menu with the other alternatives of "food", "drink", "feelings", etc., whereas every symbol generates other menus or menu hierarchies) followed by the symbols for trousers and jacket with a description of the colour of both ones. At the moment a symbol is selected, 
which, if it will be activated, leads to another menu with more clothes. At the bottom is a window with symbols, whose actions lead to a new screen ("Neustart" - start again), "undo" the last action ("Löschen" - delete), and control speech output ("OK" - speak listed) sentence. If the speech output should follow ever only after activation of one symbol, the monitor display could be even more simple, e.g. only the larger window in the middle of the just described example.

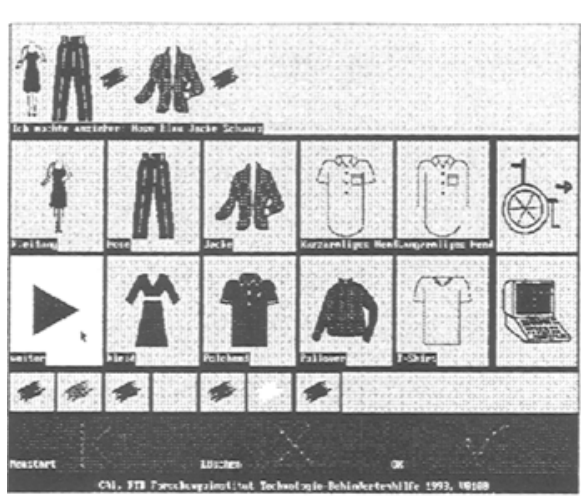

(a)

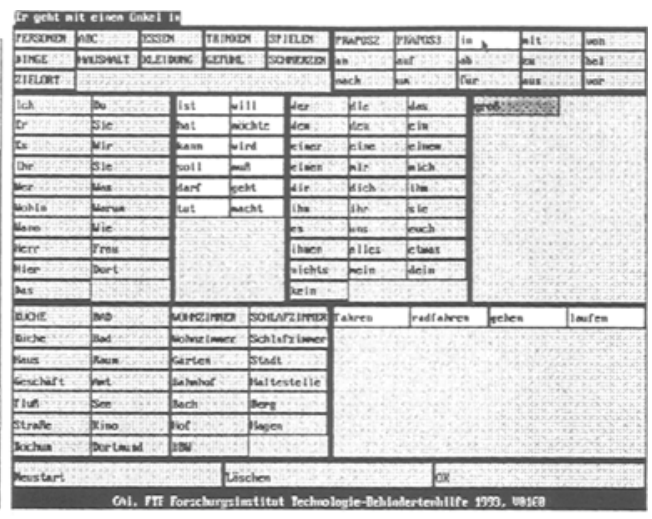

( b )

Fig. 4. Communication aids with symbols (a) and text (b)

Text-based communication aids. The same principle can be taken for communication based on text for people who can read and optionally write. Instead of symbols words, morphemes and letters are related, and the output is directed to a synthetic speech generator. A typical screen is show in figure $4 \mathrm{~b}$. At the top there is again a statement composition line. Beyond, a window allows the selection of an area of statements in order to monitor just words of current interest on the screen. A subset of the complex German grammar is built in, which permits the generation of grammatical correct statements, e.g. after choosing the subject the visible verbs are correctly conjugated. New words can be easily edited changing to a morpheme and letter screen. In comparison to pure letter editing, the efforts for the generation of statements can be much reduced after suitable individual adjustments.

Wheelchair control interface. For people with multiple and severe disabilities the continuous operation of a wheelchair with the help of a joystick is not suitable. The operation can be eased widely by using direct control in combination with digitally controlled manoeuvres. The above described interface method can be applied for initiating direct and digital control mode and for selecting and monitoring the desired manoeuvre. For reasons of safety, no communication is allowed in parallel, because wheelchair control needs continuous monitoring, but assisted communication is possible nearly at the same time as wheelchair driving and no special device and no special switch is used for communication. As already shown in figure $4 a$, a general symbol for switching to wheelchair control can be activated. Here objects are related with symbols or text and a wheelchair control activity. Figure 5a shows, as an 
example, the main menu for the choice of direct and automatic manoeuvres (like turn, side step, etc. or user recorded movements) in a scanning switch mode. At the top window the composing area for commands is found again. In the second window symbols can be chosen. Every activated symbol leads to a new menu display related to this symbol. Here a hierarchy of menus is used to display all possible manoeuvres. The wheelchair movement ends when the switch, which activates the symbols, is triggered again. Then the display shows the beginning of the menu hierarchy again.

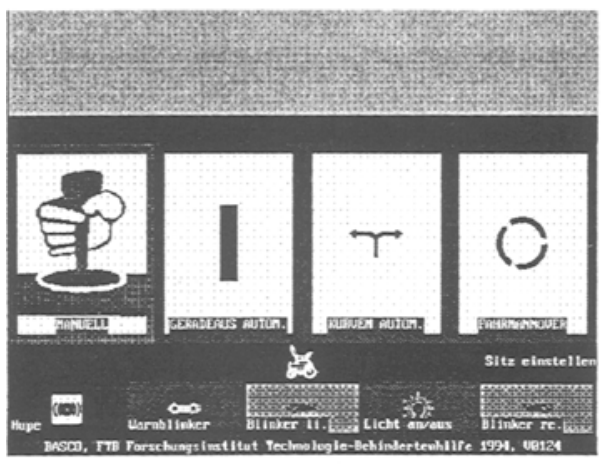

(a)



$($ ( b )

Fig. 5. Parts of control interfaces for a wheelchair (a) and environment control devices (b)

Environmental control. For those people who have problems to access directly their surroundings like doors, windows, TV, alarm, light and so on, environmental control is an important issue for independent living. Environmental objects are adressed via the same selection modes as above. They are related with symbols or text, an environmental control activity and a corresponding V/O line. Figure 5b shows a possible environmental menu, as a result of the activation of the symbol for environmental control shown in figure $4 \mathrm{a}$ (third row, last symbol).

\section{Implementation}

The user interface management system is implemented in the object-oriented programming language $\mathrm{C}++$ for IBM-compatible personal computers $(80 \times 86, \mathrm{x}>1)$, either stationary PCs for educational and training purposes (at school or rehabilitation establishments) or on portable or wheelchair-mounted notebooks.

\section{User involvement}

Before we started this project, there was a request for a versatile communication aid, which came directly from the users, teachers of the special school of our rehabilitation centre. Therefore the users were involved in the project from the very beginning. First considerations led to a small prototype for a communication aid, a 
principal demonstration of our intentions. Reviewed this, we started the development of the main software in $\mathrm{C}++$. Persons with multiple and severe disabilities tested the selection mechanisms, monitoring adjustment and different types of hierarchies. In accordance to the needs of young persons in the vocational rehabilitation process we specified the environmental control in order to assist their independent habitation. A subset of the desirable wheelchair functionality has been incorporated in the system. This will be extended furthermore. We worked out the demands for wheelchair control based on questionnaires (300 users) and interviews (50 users) [8].

\section{Perspective}

An adaptable user interface for people with special needs has been introduced. Some applications have been described for special user groups with special needs. According to these needs the interface integrates different devices. We intend to intensify the lines of communication and environmental control. At the present we integrate the wheelchair-mounted manipulator MANUS [9]. We furthermore will consider more feedback information of the system and try to get the same flexibility for system feedback as for the user input.

\section{References}

1. TIDE Pilot Action, The Synopses, March 1992, Commission of the European Communities, DG XIII

2. TIDE, 2nd phase Workplan Draft, 1992, Commission of the European Communities, DG XIII

3. Busby, G.: State of the art computer technology. ICCHP 92, Proceedings of the 3rd Intern. Conf. on Computers for Handicapped Persons, pp. 97-100 (1992)

4. H.Gabus, J.C.: JAMES - allgemeine Betrachtungen. Proc. of Congress "Technologie und Handicap", Neuchatel, June 1990, pp. 303-324 (1990)

5. McMillan, W. W.: Computing for Users with Special Needs and Models of Computer Human Interaction. ACM Conference on Human Factors in Computing Systems, CHI 1992 Conf. Proc., 3.-7. May 1992, Monterey USA, Addison -Wesley, pp. 143-148 (1992)

6. Sandhu, J.S.; Richardson, St. (1988): Concerned Technology 1989, Handicapped Persons Research Unit, Newcastle upon Tyne Polytechnic

7. Bühler, Ch.; Heck, H.: CAi - A versatile communication aid for the speech impaired. ICCHP 92, Proceedings of the 3rd Intern. Conf. on Computers for Handicapped Persons, pp. 88-96 (1992)

8. Bühler, Ch.; Schmidt, M.: User involvement in evaluation and assessment of assistive technology. ECART 2, European Conf. on the Advancement of Rehabilitation Technology (1993)

9. Kwee, H.H.: Rehabilitation robotics: softening the hardware. Intern Conf. on Rehabilitation Robotics, A.I. duPont Inst., Wilmington, Delaware (1990) 